\title{
Molecular Targets and Nanoparticulate Systems Designed for the Improved Therapeutic Intervention in Glioblastoma Multiforme
}

\author{
Authors \\ Md. Habban Akhter ${ }^{1}$, Md. Rizwanullah'르, Javed Ahmad³, Saima Amin², Mohammad Zaki Ahmad³, \\ Md. Akram Minhaj ${ }^{4}$, Md. Ali Mujtaba5 ${ }^{5}$, Javed Ali $^{2}$
}

\section{Affiliations}

1 Department of Pharmaceutics, Faculty of Pharmacy, DIT University, Dehradun, India

2 Department of Pharmaceutics, School of Pharmaceutical Education and Research, Jamia Hamdard, New Delhi, India

3 Department of Pharmaceutics, College of Pharmacy, Najran University, Kingdom of Saudi Arabia (KSA)

4 Department of Pharmacology, Maulana Azad Medical College and Hospital, New Delhi, India

5 Department of Pharmaceutics, Faculty of Pharmacy, Northern Border University, Rafha, Kingdom of Saudi Arabia (KSA)

Key words

drug delivery, pharmaceutics, cancer, anticancer drugs

received $\quad \begin{aligned} & 13.09 .2020 \\ & \text { accepted } \quad 19.10 .2020 \\ & \text { published online } \quad 10.11 .2020\end{aligned}$
Bibliography
Drug Res 2021; 71: 122-137
DOI 10.1055/a-1296-7870
ISSN 2194-9379

(c) 2020. Thieme. All rights reserved.

Georg Thieme Verlag KG, Rüdigerstraße 14, 70469 Stuttgart, Germany

\section{Correspondence}

Md. Habban Akhter

Department of Pharmaceutics

Faculty of Pharmacy

DIT University Dehradun

Uttarakhand 248009

India

Tel.: + 917983528569

habban2007@gmail.com; habban.akhter@dituniversity.edu.in

\section{ABSTRACT}

Glioblastoma multiforme (GBM) is the most aggressive and fatal CNS related tumors, which is responsible for about $4 \%$ of cancer-related deaths. Current GBM therapy includes surgery, radiation, and chemotherapy. The effective chemotherapy of GBM is compromised by two barriers, i. e., the blood-brain barrier (BBB) and the blood tumor barrier (BTB). Therefore, novel therapeutic approaches are needed. Nanoparticles are one of the highly efficient drug delivery systems for a variety of chemotherapeutics that have gained massive attention from the last three decades. Perfectly designed nanoparticles have the ability to cross BBB and BTB and precisely deliver the chemotherapeutics to GBM tissue/cells. Nanoparticles can encapsulate both hydrophilic and lipophilic drugs, genes, proteins, and peptides, increase the stability of drugs by protecting them from degradation, improve plasma half-life, reduce adverse effects and control the release of drugs/genes at the desired site. This review focussed on the different signaling pathways altered in GBM cells to understand the rationale behind selecting new therapeutic targets, challenges in the drug delivery to the GBM, various transport routes in brain delivery, and recent advances in targeted delivery of different drug and gene loaded various lipidic, polymeric and inorganic nanoparticles in the effective management of GBM. 


$\begin{array}{ll}\text { ABBREVIATION } \\ \text { BBB } & \text { Blood-brain barrier } \\ \text { BTB } & \text { Blood tumor barrier } \\ \text { CNTs } & \text { Carbon nanotubes } \\ \text { CPP } & \text { Cell-penetrating peptide } \\ \text { CSF } & \text { Cerebrospinal fluid } \\ \text { DOX } & \text { Doxorubicin } \\ \text { DTX } & \text { Docetaxel } \\ \text { EGFR } & \text { Epidermal growth factor receptor } \\ \text { FA } & \text { Folic acid } \\ \text { FGFR } & \text { Fibroblast growth factor receptor } \\ \text { GBM } & \text { Glioblastoma multiforme } \\ \text { GNPs } & \text { Gold nanoparticles } \\ \text { HER2 } & \text { Human epidermal growth factor receptor 2 } \\ \text { HGF } & \text { Hepatocyte growth factor } \\ \text { IGF } & \text { Insulin-like growth factor } \\ \text { Lf } & \text { Lactoferrin } \\ \text { MNPs } & \text { Magnetic nanoparticles } \\ \text { NLCS } & \text { Nanostructured lipid carriers } \\ \text { PDGFR } & \text { Platelet-derived growth factor receptor } \\ \text { PGDFR } & \text { Platelet-derived growth factor receptor } \\ \text { PLL } & \text { Poly(lysine) } \\ \text { PPI } & \text { Poly(propyleneimine) } \\ \text { PTX } & \text { Paclitaxel } \\ \text { RTK } & \text { Receptor tyrosine kinases } \\ \text { siRNA } & \text { Small interfering ribonucleic acid } \\ \text { SLNs } & \text { Solid lipid nanoparticles } \\ \text { Tf } & \text { Transferrin } \\ \text { TMZ } & \text { Temozolomide } \\ \text { VEGFR } & \text { Vascular endothelial growth factor receptor } \\ & \end{array}$

\section{Introduction}

GBM is a solid neoplasm represent most lethal malignancies in the brain characterized by high rate proliferation of cells and invade neighbor cells facilitated by cell migration and extracellular matrix degradation. It usually recurs after conventional therapy, such as surgical evisceration and adjuvant radio-chemotherapy. The conventional therapy results in poor efficacy and developed resistance towards chemotherapy. The biological barrier across the brain, such as BBB, BTB, and cerebrospinal fluid barrier, constitutes a significant challenge to the delivery of chemotherapeutics and biological molecules. Moreover, several clinically developed nanoparticles failed in palliating neoplasm due to the miserable cellular internalization of drug molecules from nanoparticles [1]. The new cases of brain tumor 162534 were diagnosed in males and 134317 cases in females in 2018 constituting 1.7 and $1.6 \%$ of all cancer, excluding non-melanoma skin cancer [2].

WHO reported that GBM is a malignant form of brain cancer among the various grade of brain tumors with poor prognosis and existing therapies lack efficient therapeutic efficacy. The GBM may be primary or secondary, depending upon the progress of the disease. The majority of cases arise primarily, which often constitutes more than $90 \%$ and explicates de novo in the elder age group [3]. Younger patients are more likely to endure secondary GBM devel- oped from low-grade astrocytomas. The identification basis of secondary GBM is the loss of genes in IDH1, p53, RB2 gene mutation accompanied by overexpression of epidermal growth factor receptor (EGFR), platelet-derived growth factor receptor (PGDFR), and CDK4. However, the loss of heterozygous genes from the loop of the Q10 chromosome has been identified evidently in $90 \%$ cases of GBM $[4,5]$. In contrast, primary tumors are characterized by gene alteration, p16/INK4a deletion, phosphatase and tensin homolog gene (PTEN) mutation, and EGFR amplification [6].

The treatment of glioblastoma remains a significant challenge due to its location in the intracranial space and the presence of the BBB and BTB. Therefore, there is an urgent need to develop a novel platform for the treatment of GBM to improve clinical outcomes and reduce the rate of recurrence and adverse effects. Nanoparticles based drug delivery system is one of the most promising approaches to treat GBM due to the ability to deliver a variety of lipophilic as well as hydrophilic drugs, different proteins, and peptides, various genetic materials like DNA, RNA etc., and imaging agents to the brain by crossing BBB and BTB [7-9]. Furthermore, surface modification of nanoparticles significantly improves the targeting potential and improves the accumulation of drugs to the brain, thereby enhancing the availability of the drug to the brain and reduce the extra organ toxicity of the drugs $[10,11]$.

Targeted drug delivery intended to the potential release of therapeutics in specific parts or targeted domain of the body based on enhanced permeation and retention effect (EPR) or receptor-mediated intracellular delivery and depriving the drug concentration in non-targeted parts [12]. The brain-tumor is highly challenging to cure due to the highly complex blood tumor barrier and the limited therapeutic potential of chemotherapeutics to penetrate the barrier across the brain. The growth of the brain tumor abruptly dysfunction the normal cells [13].

The potentiality of any particular substance to cross the BBB principally depends on the physicochemical properties like molecular weight, lipophilicity, and biological transport such as transport molecules, enzymes, ligands, and cell surface receptors. Furthermore, the molecular trafficking across BBB can be facilitated by passive diffusion, career mediated transport, endocytosis, and transcytosis. Several molecules have a significant role in the active transport of molecules such as ATP dependent binding cassette (ABC transporter), career mediated transport, vesicular trafficking system, p-glycoprotein, and molecular pathway mediated transport [14].

Various strategies executed in the preceding time, such as chemical drug delivery, modification on drugs manipulating the $\mathrm{BBB}$, and passive drug targeting, had inadequacy in targeting potential and results in sub-therapeutics efficacy, toxicity, variation in drug plasma profile, and safety hazards [15]. Thus, novel approaches have been employed for better drug targeting and delivering to specific regions of CNS. Different nanoparticles have been used to deliver the drugs at cellular levels through nano-fluidic channels by expending different lipidic, polymeric, and inorganic nanoparticles in the CNS.

\section{Drug Delivery Barriers to the GBM}

The trajectory barriers for nanoparticle-based drug delivery to GBM is BBB and BTB. It is comprised of various perivascular cells, endo- 
thelial cells, and microglial cells, and smooth muscle cells that enclose the brain [16]. Relating to the anatomy of the biological barrier, the lack of fenestration, the presence of tight junctions that formed an impermeable barrier ensuing confined small and lipidic molecules transport through the paracellular pathway [17]. The interaction among the components makes the barrier more complex that further prevents the entry of therapeutic molecules into the brain. Apart from that, the existence of p-glycoprotein, which acts as an efflux transporter pump out of a large number of drug molecules and creates a disturbance for penetration of compound into the brain. BBB allows selective passage of water, ion, and few lipophilic molecules and prevents the entry of large and hydrophilic drugs. Apart from paracellular transport, a transcellular pathway is involved in the transport of low molecular weight and high lipophilic compounds across the BBB [18].

Several lipophilic compounds of size < 500 Da diffuse through the endothelial membrane, while polar molecules such as protein, peptides, glucose, amino acids transport mediated through specific carrier into the cytosol after that get into extracellular spaces of the brain. However, macromolecules transported via receptormediated transcytosis, and during this process, vesicles formed which disseminate around cells eventually release their content into brain parenchyma following exocytosis. The adsorption mediated endocytosis of macromolecules triggered by the interaction of positively charged ions or molecules with the membrane phospholipid and delivered non-specifically [19]. Most importantly, BBB regulates the homeostasis process of the brain through the neurovascular unit for maintaining normal function and, at the same time, hinder drug delivery in the GBM.

The secondary barrier in brain drug delivery is BTB caused by disruption of BBB during the growth and progression of the tumor. The obstacle due to BTB for nanosized therapeutic delivery and drug penetration into the brain caused by the proliferation and growth of glial tumor cells that replace the healthy cells rapidly. The developed glial tumor leads to the growth of VEGF; angiogenesis in the hypoxic region consequently formed leaky abnormal blood vessels that conduce EPR effect. The EGFR receptor shows overexpression or the cell adhesion molecules could be the target key point [20]. In addition, BTB for targeting disease cells appears as a secondary barrier. Therefore, in brain tumor targeting, dual targeting mode is a recently established approach for targeting system to encompasses BBB (first barrier) and specific targeting to tumor cells (second barrier).

\section{Transport Routes of Therapeutic Moiety in the Brain}

\section{Extracellular route}

A small amount of circulating molecules access to CNS through the subarachnoid, plial surface, spinal nerve, cranial nerve and interfaces of the organs. Through this route, substances can reach the Virchow-Robin spaces, and hence participate in the glymphatic pathway, and are thought to be a major contributor to CNS levels of albumin. The small amount of drugs entering the brains by this pathway. However, the substances of long biological half-lives, low volume of distribution in the vascular region, and potent CNS ef- fects use the extracellular pathways as a therapeutic route. Antibodies such as IgG, IgM, erythropoietin, albumin, and modified lysosomal enzymes are thought to enter the BBB-intact CNS by these routes [21].

\section{Trans-endothelial diffusion}

By diffusion, the only a small molecule could enter the brain through a non-saturable mechanism. The diffusion through BBB required traversing the entire cell, unlikely to passage through the cell membrane. The degree of the traverse is depended mainly upon the physicochemical properties of substances such as solubility, molecular weight, and bonding level. In the CNS, partitioning of biological molecules largely favored by lipophilicity. The ideal ratio of octanol/water partition for brain extraction lies in between 10:1-100:1. The surface charge of aqueous and lipid environment cerebrospinal fluid (CSF) can influence the partitioning behavior. The uptake of weak bases is slightly favored in the acidic environment of CSF and vice-versa [22].

\section{Carrier mediated transport}

This mechanism is the bidirectional transport of biological molecules across the brain to blood and vice-versa and maintain homeostasis. It has substantial control over both efflux and influx phenomena for selective transport of molecules in circulation between blood and brain and vice-versa. The smaller molecules $<500 \mathrm{Da}$, the destination to the brain dominated by passive transcellular diffusion and efflux controlled by $A B C$ transporter such as P-gp and MDRP (multi-drug resistance protein) [23]. The precise modification of carrier proteins leads to the translocation of constructs into the cytosol.

\section{Receptor-mediated transcytosis}

This mechanism admits the transport of large number cargoes due to potential targeting capability. The nanoparticles would target the receptor with abiding by imputes of a high level of receptor expression in the specific brain vasculature (luminal side) compare to other tissues. Additionally, the receptor-mediated transcytosis has high turnover, and broad substrate recognition and characteristics of the transporter are easily modified [24].

There are many receptors present abundantly in the BBB for which receptor-based transcytosis phenomena of drug delivery is well suited. These receptors include low-density lipoprotein, insulin, leptin, integrin, and transferrin. The various probable routes for the transport of foreign substances across BBB are shown in >Fig. $\mathbf{1}$.

Low-density lipoprotein, transferrin, insulin, and leptin The low-density lipoprotein is well known and extensively canvassed receptor in the transport and signaling of peptides and protein delivery across BBB. The protein ligands for this receptor are ApoE, ApoB fragments, and Angiopep-2. Transferrin has a high expression level and well-characterized among BBB transport receptors in BBB and widely distributed in brain endothelium and brain receptors. Moreover, integrin receptors have not been dominantly present in the microvasculature of the brain, preferably been employed for the extensive potential targeting to the brain tumor and ablaze region of the brain. Of the various type of integrin, $\alpha_{v} \beta_{3}$ is highly overexpressed in neovasculature for which substrate ligand is a cyclic peptide. Despite 


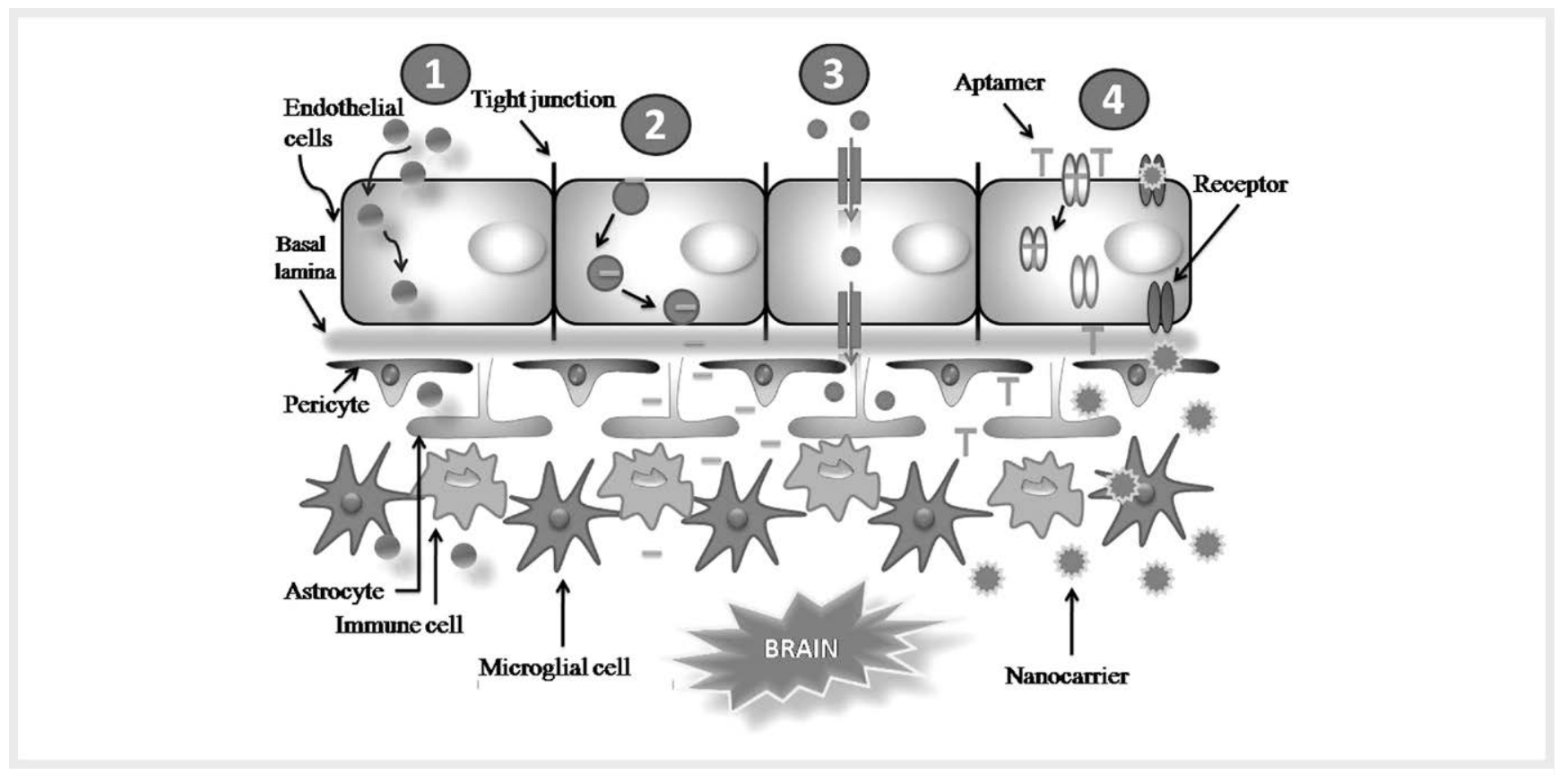

- Fig. 1 Different probable routes for the transport of foreign substances across the blood-brain barrier.

receptor-mediated drug delivery in the brain, various other ion channels play a significant role in brain delivery along with recycling and intracellular eliciting capability [25-27].

Angiopep-2 is a versatile sequence of aprotinin with other human proteins with Kunitz domain that interacts with low-density lipoprotein, especially LRP1, widely exploited for the transport of small chemotherapeutic agents viz., paclitaxel, and doxorubicin [28]. However, the applicability of Angiopep-2 with such anticancer drugs for the treatment of recurrent high-grade glioma along with antibodies trastuzumab is in a clinical trial [29].

\section{Adsorptive-mediated endocytosis}

TAT peptide is the most common example of protein delivery into CNS using nanoparticles. Due to the lack of selectivity in brain targeting, limited data are available in the public domain regarding intact BBB penetration. However, a recent study addressed the brain accumulation of therapeutics, the improved therapeutic effect for the intended targeting shuttle in the brain endothelium. The most common example is ritonavir loaded TAT-peptide nanoparticle improved 800 folds delivery in the brain. As expected, TAT nanoparticles were highly internalized in the brain, such as capillary endothelial cells due to adsorption interceded endocytosis and drug released tardily, further substantiated by parenchyma/capillary ratio [30]. Nevertheless, another example is dual-targeted liposomes based on Angiopep-2-oligoarginine, outperformed with single targeting peptide both in vitro as well as in vivo [31].

\section{Molecular Targets in GBM}

The Cancer Genome Atlas Research study investigated that signaling pathways have a significant role in the development of brain tumors and the progress of many diseases. The RTK/Ras/PI3K sign- aling pathway contributed upto $90 \%$ of cases in tumerogensis and other cases pRB signaling pathway reported in the development of GBM [32]. Several molecular pathways involved in tumerogenesis process and therapeutic target in GBM are given below [33].

\section{Ras/MAPK/ERK and Ras/PI3K/AKT pathway}

Receptor tyrosine kinases (RTKs) are a family of cell surface receptors, to which a number of ligands likewise, cytokines, hormones, growth factors, and various extracellular signaling molecules bind. The binding of the ligand with such receptor leads to downstream signaling cascades Ras/MAPK/ERK and Ras/PI3K/AKT [34]. These pathways control the regulation of cell proliferation, survival, differentiation, and angiogenesis. Tyrosine kinase receptors are essentially comprised of an extracellular ligand-binding domain, an intracellular tyrosine kinase domain, and a hydrophobic transmembrane domain. The activation of the receptor results in dimerization accompanied by the autophosphorylation of the tyrosine kinase domain. The cellular events further lead to activation of downstream signaling pathways, i.e., Ras/Raf/MEK1/2/ERK1/2 and Ras/ $\mathrm{PI} 3 \mathrm{~K} / \mathrm{AKT}$ has a prominent role in cell proliferation, survival, invasiveness, and angiogenesis process [35]. Therefore, RTK is a promising therapeutic target in GBM therapy. Some of the receptors belonging to this family are briefly discussed below.

\section{EGF receptor}

The EGF family is divided into four class of receptors; EGFR/ErbB-1/ HER1, ErbB-2/ human epidermal growth factor receptor 2 (HER2)/ Neu, ErbB-3/HER3, and ErbB-4/HER4. The gene mutation and amplification of HER 1 receptor were detected in $40-60 \%$ of cases of GBM contemplated. A truncate mutated variant III EGFR, i. e., EGFRVIII dominantly expressed in GBM [36, 37]. 


\section{VEGF receptor}

It is a potent angiogenic protein commonly known for enhancing vascular permeability. The microenvironment of the brain tumor has a hypoxic condition, which later induces hypoxia transcription factor, and their translocation inside the nucleus leads to VEGF gene activation, in turn, enhance the angiogenesis to antagonize hypoxia. The GBM tumor has a high expression level of VEGF, which imparts the leaky vasculature in tumor sites [36, 37].

\section{PDGFR}

The signaling cascade is related to the growth and development of healthy tissues, and dysfunction leads to oncogenesis. TCGA research analyzed the amplification of platelet-derived growth factor receptor alpha (PDGFR $\alpha$ ) in $10-13 \%$ of the cases of GBM reported [36, 37].

\section{PI3K/AKT/mTOR pathway}

The PI3K/AKT/mTOR pathway is activated by tyrosine kinase growth factor receptor (G-protein receptor), as shown in > Fig. 2. Accompanying activation PI3K translocates to the plasma membrane resulting formation of phosphatidylinositol 3,4,5-triphosphate (PIP3), which further stimulates serine/threonine kinase phosphoinositide-dependent kinase 1 (PDK1) and AKT (at threonine 308) [38]. Phosphatase and tensin homolog (PTEN) antagonizing the process by removing the phosphate group from PIP3 to PIP2 [39]. The activated AKT phosphorylates FOXO family resulted in the suppression of transcription of pro-apoptotic protein such as BAD and GSK3 [39]. The mTOR works by two complex forma- tions, mTORC-1 and mTORC-2, linked with growth factor cascades with protein translation, growth of cells, proliferation, and survival. The mTORC 1 acts as a downstream activator, whereas mTOR2 upstream activator of PI3K/AKT signaling pathway. In mTORC1, mTOR acts, together with PRAS40, RAPTOR, and mLST-8/GßL. mTOR linked with RICTOR, mSIN-1, and mLST-8/GßL, forms the mTORC2 complex. mTOR has significant implications in cell proliferation in GBM associated with activation of S6K1 and, in turn, S6 [40]. The medical prognosis reported active state of mTOR in GBM leads to the upregulation of several proteins involved in cell cycle progression or metabolism, finally affirming tumorigenesis. In contrast, mTORC-2 activity has also been shown to be elevated in glioma cell lines and clinical GBM isolates [41].

\section{p53 protein pathway}

Many types of cancer growth reported alteration in p53 pathway, including GBM. Cancer Genome Atlas Research Network founded upto $35 \%$ of cases in GBM p53 mutated. The p53 protein signaling pathway involved in the activation of genes linked with apoptosis due to cell cycle arrest. The stress signal elicits p53 response and activates p21 gene that inhibits $C d k 2 / 4$ resulting in inhibition of cell cycle progress [42].

\section{pRB pathway}

This pathway plays a significant role in the suppression of cell cycle through interaction with E2F, transcription factors, and $75 \%$ of the cases reported with pRB signaling alteration due to genetic mutation or deletion in GBM. Palbociclib is an inhibitor of the PRB gene

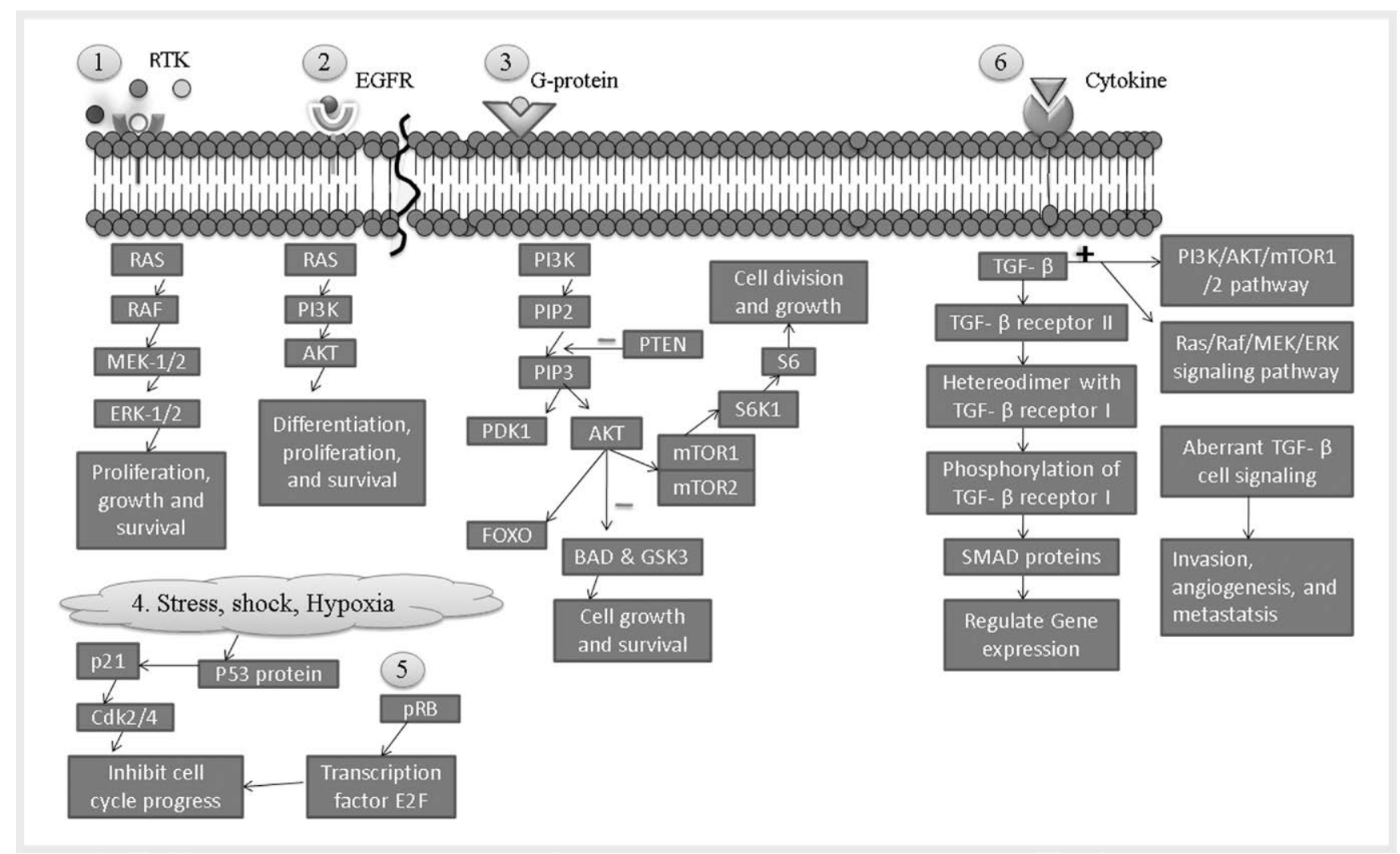

Fig. 2 Schematic representation of molecular pathways involved in the development of GBM. 
has been shown as a growth inhibitor in the intracranial GBM xenograft model [43].

\section{TGF- $\beta$ signaling pathway}

TGF- $\beta$ belongs to the cytokine class has many potential effects on cell related growth, immunity, and cell death. It binds with receptor II of TGF- $\beta$ that leads to the formation of heterodimers TGF- $\beta$ receptor I and phosphorylation of TGF- $\beta$ receptor I and finally, activation of SMAD proteins that regulate the gene expression [44]. TGF- $\beta$ signaling also activates Ras/Raf/MEK/ERK and PI3K/AKT/ mTOR signaling pathway [45]. TGF- $\beta$ signaling is associated with inhibiting cell proliferation because of tumor suppressor function. The deviation in substantial norms of this pathway leads to invasion, inflammation, and angiogenesis and metastasis [46].

\section{Other Signaling Pathway Involved in GBM}

\section{c-MET/HGFR pathway}

Hepatocyte growth factor (HGF) is a ligand for activation of HGFR/ c-MET pathway, which is found in nearby brain tumor cells. This pathway individually accounts for $2-4 \%$ of the generation of brain tumor and is mainly activated in high-grade mesenchymal subtypes of GBM. It regulates multiple cell function proliferation, motility, and survival. The aberrant activation due to gene amplification or mutation is associated with prompt tumor growth, invasion, and angiogenesis [47]. The c-MET pathway is predominantly activated in high-grade GBM due to elevated levels and mutation in the promoter region of HGF. Thus, targeting c-MET could be a novel approach in the apoptosis of high-grade GBM cells [48].

\section{Fibroblast growth factor receptor (FGFR) pathway}

The chromosomal amplification, mutation, translocation and aberration in human fibroblast growth factors receptor found in human lung cell carcinoma and breast carcinoma. However, 3.2\% cases of mutation in this receptor linked with GBM due to fusion between the coding sequence of the tyrosine kinase domain of the FGFR gene with TACC 3 and TACC1 gene associated with mitotic spindle localization $[49,50]$. This receptor is involved in the growth of cultured GBM cell lines and blockade of FGFR signaling by RNA interference and inhibits the growth of GBM cell proliferation. It was reported that FGFR1 expression was reduced in chemotherapy-treated GBM patients due to poor predictive marker survival and tumor progression. Currently, the FGFR1 pathway is implicated in the radiosensitivity of GBM, both in vitro and in vivo. GBM cell lines expressing FGFR1 along with shRNA and siRNA to silence FGFR1 both in vitro and in vivo settings. Further, 2Gy irradiation analysis was shown that inhibition of EGFR1 enhanced the radiosensitivity of both the cell lines [51].

\section{Insulin-like growth factor (IGF) pathway}

This pathway is a complex network system that includes IGF1-IR, IGFII- IIR, high-affinity binding proteins such as IGFBP-1 to IGFBP6 , and their ligand IGFI, and IGFII. The expression level of IGF1-IR in GBM is associated with shorter survival and lowered response to temozolomide, suggesting the signaling pathway of IGF-1R receptor in GBM tumerogensis [52].

\section{Calcium signaling pathway}

The calcium ion influx either from the external environment or internal sources causes a tremendous increase in calcium concentration in the cytoplasm, which has been widely utilized for signal transduction. The calcium signaling channel has a significant level of control over tumorigenesis process in GBM, and cell proliferation, quiescence, migration, and gene transcription. Glioblastoma development is thought to be implicated in many co-opting pathways which directly involved in the organ system formation. The deregulation of the normal calcium ion signaling elements contributes to the progression of the disease. Calcium influx is mediated by voltage-gated calcium ion channel. Of the various types of calcium ion channels, the aberrant T-type channel has been implicated in GBM cell progress and proliferation [53]. A recent study explored membrane depolarization of calcium channel blocker speed up programmed glioma cell death by worsening mineral, protein, amino acid, and essential nutrient transport inside cells. Zhang et al. studied RNAi-mediated attenuation of the calcium signaling pathway, or mibefradil treatment was efficient to suppress the growth, the survival of GBM tumor, and also sensitized them to temozolomide chemotherapy. Proteomic and transcriptomic investigation revealed that calcium channel inhibition altered cancer signaling pathways and gene transcription. The inhibition of calcium channel suppressed GBM stem-like cells partly by inhibiting prosurvival AKT/mTOR pathways and as well as stimulating proapoptotic survivin and BAX pathways. In addition, inhibition of these depletes oncogenes expression of PDGFA, PDGFB, and TGFB1 and increased expression of tumor suppressor genes (TNFRSF14 and HSD17B14) [54].

\section{cGMP/PKG pathway}

The cGMP is an intracellular messenger II capable of mediating action NO and natriuretic peptides, thus regulating a broad array of physiologic and biological processes. The elevated cGMP level exhibits their physiological action predominantly mediated by cGMPdependent protein kinase (PKG). PKG activation leads to the lowering of cytosolic calcium ion and reduced sensitivity of myofilaments towards calcium ion, consequently resulting in smooth muscle relaxation [55]. Recently, it has been established that low physiological concentration of NO acts as an anti-apoptotic/prosurvival factor in specific neural cells, e. g., PC12 cells and motor neurons. The anti-apoptotic activity of NO is mediated partly by cGMP pathway via downstream target protein, PKG. Inhibition of soluble guanylyl cyclase and reducing the level of cGMP cause apoptosis in unstressed neural cells such as N1E-115 cells NG108-15. Therefore, cGMP/PKG pathway seems to play an indispensable role in preventing the activation of a pro-apoptotic pathway, thus promoting neural cell survival $[55,56]$.

\section{Nanoparticles Based Approaches in Glioblastoma Treatment}

Many researchers in their investigations documented the significant impact of nanotechnology in designing of nanoparticles/surface-engineered nanoparticles to explore the existing therapeutic modalities with improved efficacy in GBM. Different nanoparticles 
exploited for the effective management of GBM in preclinical investigations are illustrated in > Fig. 3.

\section{Lipidic nanoparticles}

Liposomes

Liposomes are nanosized vesicles that are formed by encasing aqueous inner core followed phospholipid bilayer. Both lipophilic and hydrophilic drug molecules can be entrapped into liposomes in the lipid bilayer membrane, depending upon the categorized layer for therapeutics. Lipid soluble drugs get entrapped within the bi-layered lipidic membrane, while hydrophilic drugs get entrapped in the central aqueous core of the vesicles $[57,58]$. Liposomes are a potential carrier for controlled drug release of tumors therapeutic agents, antibiotics, for gene and antisense therapy through nucleic acid sequence delivery.

Liposome has been widely investigated for CNS delivery of chemotherapeutic agents due to biocompatibility and biodegradability in vivo. However, conventional liposomal delivery faces hindrances in drug delivery owing to the process of opsonization, rapid clearance from the macrophagic phagocytic system. The circulation time could be enhanced by PEGylation technique; however, it reduced the transport rate of biomolecules. To circumvent such limitation and the possibility of enhancing drug targeting to CNS, conjugation of liposome with ligands is an ultimate option for the precise delivery of bioactive agents [59].

The functionalized liposomes with active ligand enhance the targeting potential due to improved BBB crossing and approaching to the brain tumor. In this context, Chen et al. developed doxorubicin (DOX) loaded liposomal system with several ligand molecules and assessed the targeting capability of across BBB [60]. The ligands employed in his study were angiopep-2, T7, c(RGDfK), Pep- tide-22, D-SP5, and Pep-1. The cell uptake study revealed peptide-22, and c(RGDfK) decorated liposomes were shown synergistic effect and significantly increased the internalization of $\mathrm{li}$ posomes on $\mathrm{U} 87$ cells and thus selected to fabricate the BBB/BBTB double-cross, glioma targeting liposomal delivery system c(RGDfK)/ Pep-22-DOX-liposome. Furthermore, the in vivo imaging study of c(RGDfK)/Pep-22-liposome demonstrated increased brain tumor distribution compare to liposomes modified with a single ligand. The result interpreted the mean survival time of glioma-bearing mice was 39.5 days longer after treatment with c(RGDfK)/Pep22-DOX-liposome than those treated with plain DOX or other controls. In another study, transferrin (Tf) decorated DOX-loaded cell penetrating peptide (CPP) decorated sterically stabilized liposome (Tf-CPP-SSL) was fabricated to ameliorate the therapeutic effect in gliomas [61]. The mechanistic approach is based on both identifications of Tf receptors over the surface of BBB followed by internalization in glioma cells and, finally, the lysosomal escaping potentiality of CPP. These liposomes had the highest targeting efficacy for brain microvascular endothelial cells and C6 cell uptake but avoided capture by healthy cells. In addition, fluorescence resonance energy transfer technology and co-culture models of BBB and glioma C6 cells demonstrated that Tf-CPP-SSL was transported across the BBB without rupture of liposomal membrane or drug leakage, or cleavage of the ligand. Tf-CPP-SSL potentially crossed the BBB and finally entered into glioma C6 cells. Furthermore, confocal microscopy investigated that Tf-CPP-SSL was entrapped in the late endosomes of glioma C6 cells and then escaped from lysosomes successfully to release the liposomal contents into the cytosol. Afterward, the chemotherapeutic entrapped agent, DOX, entered the nucleus to exhibit enhanced pharmacological action. Similarly, Lakkadwala et al. developed DOX and erlotinib co-loaded

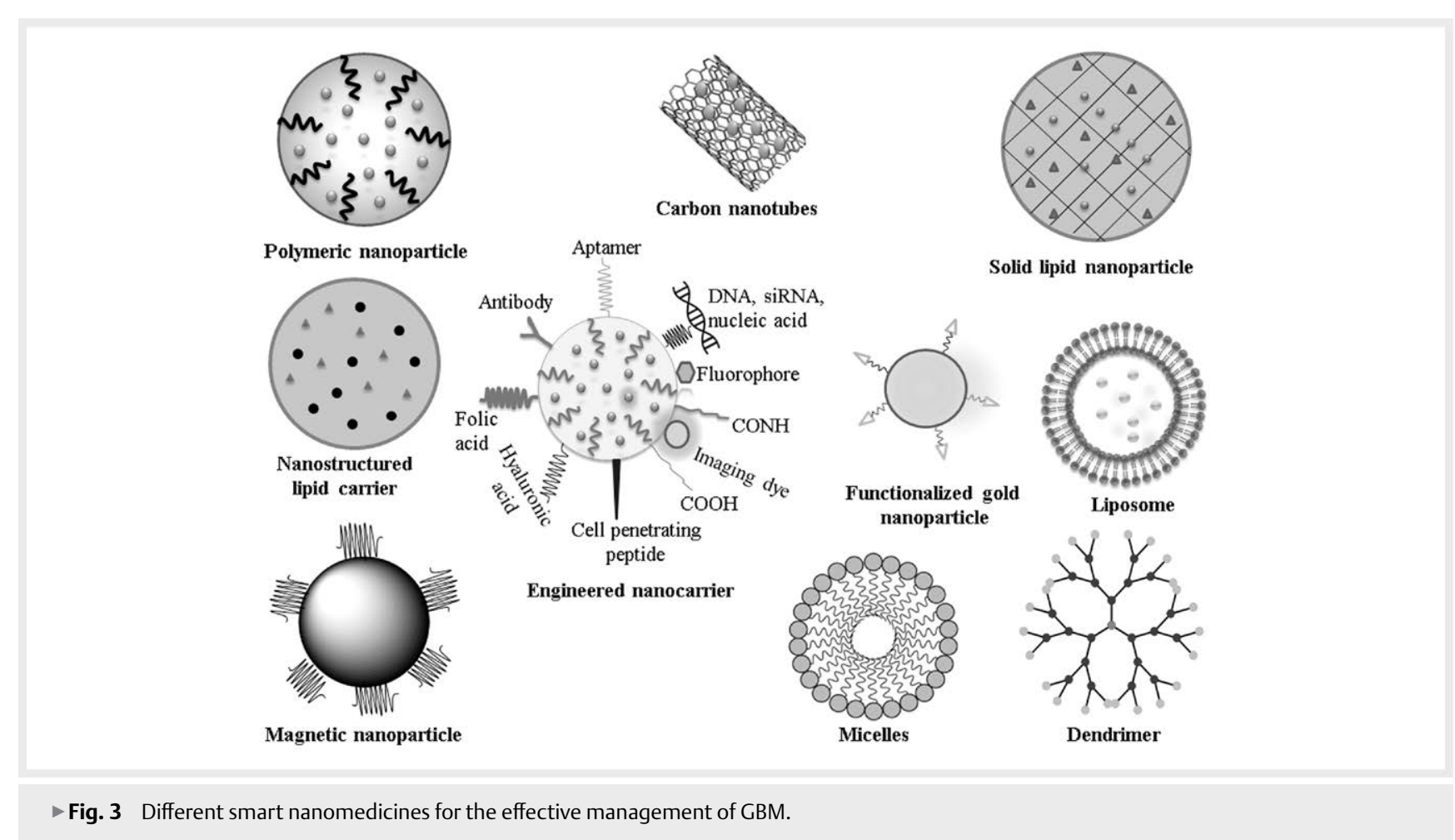


Tf decorated liposomes meant for receptor-mediated transcytosis and conjugated with cell-penetrating peptide for deeper penetration into glial tumor [62]. The fabricated system was satisfactorily biocompatible, indicate in cytotoxicity and hemocompatibility studies. The developed dual drug-loaded liposomes to enhance their translocation across the BBB to GBM. The study further suggested co-delivering DOX and erlotinib from Tf-Pen liposomes significant translocation ( $15 \%$ ) around the co-culture endothelial barrier led to inhibition of tumor growth in a brain tumor model in vitro. The biodistribution revealed the effective accumulation of DOX and erlotinib 12 and 3.3 fold higher from Tf-Pen liposomes in mice brain compare to free drugs. Moreover, Tf-Pen liposomes exhibited excellent anti-tumor efficacy by reducing $90 \%$ tumor in mice and increased median survival time.

\section{Solid lipid nanoparticles (SLNs)}

Solid lipid nanoparticles (SLNs) as nanocargo have shown significant improvement in drug permeability across BBB. It is a biodegradable and biocompatible nanoplatform that has the capability to entrap both hydrophilic and lipophilic drugs [63]. SLNs offers several advantages such as flexibility in the surface modification in concern to both charges and particle size. Nanotechnology platform provides drugs incorporated in SLNs that improved the permeability of BBB [64]. In a study, surface-functionalized SLNs were developed using apolipoprotein $\mathrm{E}$ for improved delivery via receptor-targeted delivery in the brain [65]. The surface modified SLNs binds with low-density lipoprotein receptors lies on the endothelial cells of BBB. The MTT and LDH assays in the human cerebral microvascular endothelial cells (hCMEC/D3) cell line, a human BBB model, revealed no toxicity up to $1.5 \mathrm{mg} / \mathrm{ml}$ over $4 \mathrm{~h}$ of incubation. The brain permeability was appraised in transwell devices with monolayers of hCMEC/D3 and affirmed 1.5-fold increment brain permeability for functionalized nanoparticles over non-functionalized ones. Garanti et al. developed asiatic acid (AA) encapsulated RGD-modified SLNs (AA-RGD-SLNs) for targeted treatment of GBM [66]. The developed AA-RGD-SLNs showed significantly improved cellular internalization and cytotoxicity of AA against U87-MG cells compared to non-targeted AA-SLNs and AA alone. Furthermore, in vivo study in spheroid models revealed significantly enhanced tumor growth inhibition and spheroid penetration of AA-RGD-SLNs compared to non-targeted AA-SLNs and AA alone. In another study, Rahiminejad et al. fabricated indirubin (IN) encapsulated SLNs (INSLNs) for effective management of GBM [67]. The developed INSLNs exhibited sustained release of IN in both normal brain $\mathrm{pH}$ of 7.2 and tumoric $\mathrm{pH}$ of 6.8. Furthermore, IN-SLNs exhibited significantly improved cellular uptake and cytotoxicity against U87-MG cells compared to free IN and blank SLNs.

Nanostructured lipid carriers (NLCs)

NLCs overcomes the limitation of SLNs such as crystalline solid lipid phase, drug expulsion during storage, low payload. NLCs are considered as a second-generation SLNs comprised of solid lipid blends in oil (liquid lipid) evolved as an alternative strategy for drug delivery application. NLCs acquire better inclusion of drugs inside the lipidic matrix and achieve high entrapment efficiencies [68, 69]. PEGylated NLCs remain a long time in the systemic circulation due to un-captured in macrophages than conventional NLCs. It has the tendency to traverse leaky vasculature and passively accumulated near cancer cells depending upon enhanced permeation and retention effect. Additionally, surface modification of NLCs with a ligand such as an antibody, proteins, or peptides or other active biomolecules has been an attractive promising approach for therapeutic utility in cancer [70]. In this context, Song et al. developed arginineglycine-aspartic acid peptide (RGD) functionalized temozolomide (TMZ) loaded NLCs (RGD-TMZ-NLCs) for improved efficacy against GBM [71]. In the in vitro cell line against U87MG cells, RGD-TMZNLCs exhibited 2 and 10 folds reduction in $\mathrm{IC}_{50}$ value compared to non-targeted TMZ-NLCs and TMZ solution respectively. Moreover, in vivo studies in the nude $B A L B / c$ mice revealed the highest tumor inhibition efficacy in comparison with non-targeted TMZ-NLCs and TMZ solution. In another study, Zhang et al. formulated dual ligand \{lactoferrin (Lf) and RGD\} functionalized, TMZ, and vincristine (VCR) co-loaded NLCs for better management of GBM [72]. The developed dual ligand decorated dual drug loaded NLC exhibited the highest cellular uptake and cytotoxicity against U87 MG cells compared to single ligand decorated NLCs, non-targeted NLCs, and pure TMZ and VCR solutions. Moreover, dual ligand decorated dual drug-loaded NLC exhibited the highest tumor tissue distribution and tumor growth inhibition in U87 MG cells bearing nude BALB/C mice compared to single ligand decorated NLCs, non-targeted NLCs, and pure TMZ and VCR solutions. Furthermore, Chen et al. developed curcumin (CUR) encapsulated NLCs (CUR-NLC) for the management of GBM [73]. The developed CUR-NLCs exhibited significantly higher cellular uptake and 4 fold reduction in $\mathrm{IC}_{50}$ against A172 brain cancer cells compared to CUR solution. Moreover, CURNLCs showed 6.4 fold higher CUR accumulation in the brain and 4.2 fold higher brain tumor inhibition efficacy in nude mice bearing A172 xenograft compared to CUR solution.

\section{Polymeric nanoparticles}

Polymeric nanoparticles

Polymeric nanoparticles are fabricated from the natural or synthetic polymer of biologically safe in vivo. The general size ranges from 1-1000 nm depending upon the drug delivery requirement based on different viable routes. The therapeutics molecules can be incorporated throughout the surfaces of the polymeric matrix, referred to nanosphere or inside the polymeric core called nanocapsule [74-76]. Nanoparticle mediated therapeutic delivery to target prone sites largely depends on the physical characteristics of nanoparticles such as particle size, shape, surface charge, and surface modification. Despite these, it offers multiple delivery benefits as non-interaction with normal body cells, reduced toxicity, stealth properties due to long circulation time, enhanced accumulation and retention effect, enhance cellular uptake, uniform distribution to organs, biodegradability, biocompatibility, high payload, rationale, and controlled drug delivery over other colloidal systems $[77,78]$. In a study, Ramalho et al. developed OX26 type monoclonal antibody (mAb) decorated TMZ loaded PLGA nanoparticles (OX26-TMZ-PLGA-NPs) for Tf receptor-targeted delivery of TMZ in the management of GBM [79]. Their results showed that the developed OX26-TMZ-PLGA-NPs exhibited significantly enhanced cellular uptake and cytotoxicity against U251 and U87 compared to non-targeted TMZ-PLGA-NPs and TMZ solution. Similarly, Li et al. developed Lf decorated shikonin (SHK) loaded PLGA nanoparticles 
(Lf-SHK-PLGA-NPs) for the management of GBM [80]. In vitro cell line study in C6 cells revealed significantly higher cytotoxicity and showed 1.2 and 1.43 fold reduction in $\mathrm{IC}_{50}$ value compared to nontargeted SHK-PLGA-NPs and SHK solution, respectively. Moreover, in vivo studies revealed significantly higher brain concentration and brain bioavailability of Lf-SHK-PLGA-NPs compared to SHK-PLGANPs and SHK solution. In another study, de Oliveira Junior et al. developed melatonin (MLT) loaded polycaprolactone $(\mathrm{PCL})$ nanoparticles (MLT-PCL-NPs) for intranasal delivery of MLT for the management of GBM [81]. In vitro cytotoxicity study in U87MG cells revealed strong efficacy and showed almost 2500 fold reduction in $\mathrm{IC}_{50}$ value compared to MLT solution. Moreover, MLT-PCL-NPs exhibited 7.6 fold improved absolute bioavailability in male Wistar rats compared to MLT suspension after intranasal administration.

\section{Polymeric micelles}

Micelles comprised of amphiphilic block copolymer in contact with aqueous phase they aggregate form stable spheroid like nanostructures. The micelles possess a hydrophobic core and hydrophilic surface, thereby enhancing the possibility of poorly aqueous soluble substance in the hydrophobic core. Polymeric micelles offer potential opportunities to improve delivery to the brain tumor and have been largely explored for the delivery of the chemotherapeutic agent, genes, proteins, and antibodies in brain tumors. Despite these, micelles surface modification, ligand-oriented drug delivery, and trigger dependent release mechanism design particularly delivered site-specific delivery of therapeutics has added advantages $[82,83]$. In this context, Quader et al. developed CRGD decorated epirubicin (Epi) encapsulated micelles for effective management of GBM [84]. The developed targeted micelles showed significantly improved cellular uptake in U87MG cells compared to non-targeted micelles and pure drug solution. Moreover, cRGD-Epi micelles exhibited significantly higher growth inhibition compared to non-targeted micelles and Epi solution due to the integrin-mediated targeted delivery of Epi in the GBM. Similarly, Li et al. developed cRGD decorated docetaxel (DTX) encapsulated polylactic acid-polyethylene glycol (PLA-PEG) micelle for the management of GBM [85]. The developed CRGD decorated micelles exhibited significantly enhanced cellular uptake and cytotoxicity in U87MG cells compared to non-targeted micelles and pure DTX. In vivo biodistribution study in BALB/c mice showed significantly enhanced GBM tissue uptake due to $\mathrm{Tf}$ receptor-mediated uptake after intravenous (i.v) delivery in nude BALB/c mice compared to non-targeted micelles and pure DTX. Moreover, CRGD decorated micelles exhibited significantly higher anti-glioma efficacy compared to non-targeted micelles and pure DTX in U87MG tumor-bearing BALB/c mice. In another study, Huang et al. developed CRGD decorated DOX and paclitaxel (PTX) co-encapsulated pluronic micelles in the management of GBM [86]. cRGD-DOX-PTX-micelles exhibited significantly higher transport rates across the in vitro blood-brain barrier model, cellular internalization, cytotoxicity, and apoptosis against U87 MG cells compared to non-targeted DOX-PTX-micelles and native drugs. Further, CRGD-DOX-PTX-micelles exhibited significantly higher tumor accumulation and tumor inhibition rate compared to non-targeted DOX-PTX-micelles and native drugs. Moreover, almost negligible major acute toxicity was observed after i. v. administration of CRGD-DOX-PTX-micelles.

\section{Dendrimers}

Dendrimers are highly branched, synthetic polymeric macromolecules with various enlargements. The size ranges from a few nanometers to $100 \mathrm{~nm}$. It is chemically synthesized with controlled polymeric reaction, their surface tuning and biodegradability can be adjusted. The large surface area available for multiple surface functionalization, high loading capacity, reduced clearance from the reticuloendothelial system, and a safe biological carrier for therapeutic delivery of large number biological micro or macromolecules $[87,88]$. The extensive application of dendrimers for the delivery of chemotherapeutic agents and genes for the treatment of GBM and other malignant brain tumors has been numbered in the literature. Swami and associate targeted sigma receptor of the brain by pHBA-dendrimer conjugates as a colloidal carrier for site-specific targeting of DTX [89]. Moreover, the cytotoxic study, cellular uptake study was performed against U87MG human glioblastoma cells, formulation exhibited considerable anticancer effect compared to the plain drug. Further, pHBA-dendrimer conjugates increased brain uptake of DTX, confirmed by a high recovery rate compared with unconjugated dendrimers or marketed formulation Taxotere ${ }^{\circledR}$. Similarly, Sharma et al. developed chitosan decorated PAMAM dendrimers for improved delivery of TMZ in the management of GBM [90]. The developed dendrimer exhibited significantly enhanced cellular internalization and cytotoxicity against U-251 and T-98G glioma cells compared to free TMZ. Moreover, dendrimers exhibited significant brain concentration, and almost 1.5 fold improved half-life compared to free TMZ. In another study, Gajbhiye and Jain developed polysorbate 80 (P80) anchored DOX loaded poly(propyleneimine) (PPI) dendrimers for GBM treatment [91]. The developed P80 anchored PPI dendrimers exhibited significantly enhanced cellular uptake and cytotoxicity against U87 MG cells compared to without P80 anchored PPI dendrimers and free DOX. Moreover, P80 anchored PPI dendrimers exhibited a significantly higher concentration of DOX in the brain, thereby improved survival rate in U87MG tumor-bearing male Sprague Dawley xenografts compared to without P80 anchored PPI dendrimers and free DOX.

\section{Inorganic nanoparticles}

\section{Gold nanoparticles}

Gold nanoparticles (GNPs) are used in different scientific domains, of which the biomedical field is most extensively using GNPs system for the effective delivery of chemotherapeutics and genetic materials in the management of GBM. They have favorable properties like improved stability, excellent compatibility, low toxicity [92]. In this regard, Kong et al. used GNPs as a siRNA carrier for efficient transfection of BCL2L12 siRNA to U87MG cells for a reduction in BCL-2 protein levels [93]. The developed siRNA conjugated GNPs consisted of nanostructure of densely packed siRNA molecules and showed a $22 \%$ reduction in BCL-2 proteins level compared to the native genetic material. In another study, Kouri and associates proved that NPs could cross the BBB by executing work on miR182-based gold-cored spherical nucleic acid NPs on U87 cells and patient-derived glioma-initiating stem-like cells (GICs) [94]. In vitro results expressed that a decrease in miR182 target genes BCL2L12 and c-Met protein levels. The in vivo results showed similar findings in GIC and U87MG-derived brain xenografts. Furthermore, Zhao 
and colleagues designed and developed multifunctional polyethylenimine-entrapped GNPs for target-specific SPECT/CT imaging as well as radionuclide therapy of glioma [95]. Polyethylenimine was used for modification with polyethylene glycol (PEG), gliomaspecific peptide (chlorotoxin, CTX), and 3-(4-hydroxyphenyl) propionic acid-OSu (HPAO), and further, entrap GNPs. After ${ }^{131}$ I radiolabeling through HPAO, the ${ }^{131}$ I-labeled CTX-functionalized GNPs was developed as a nanoprobe for glioma targeting. The radio-labeled GNPs established high stability and radiochemical purity and an excellent nanoprobe for the targeted SPECT/CT imaging and radionuclide treatment in GBM both in vitro and in vivo. The NPs due to unparalleled modification via CTX, the generated nanoprobe showed potential permeation across BBB was for specific targeting glioma cells in an intracranial glioma rat model.

\section{Magnetic nanoparticles}

The response of magnetic nanoparticles (MNPs) towards the external magnetic field, making ideal carriers in drug delivery and medical imaging. MNPs can be fabricated in such a way by an external magnetic field to be utilized for various applications, including cancer therapy and magnetic resonance imaging. The MNPs are essentially comprised of the inner magnetic core of some metal oxide and outer functional components. In the context of glioma tumor therapy, superparamagnetic iron oxide NPs induced hyperthermia that elevates immunogenic response to cell death by induction of altering magnetic field is predefine in literature. Thermo-responsive MNPs is an ideal carrier for a dual purpose, such as hyperthermia followed by additive therapeutic effect in cancer treatment $[96,97]$. Lu and associates developed cetuximab (C225)-encapsulated core-shell $\mathrm{Fe}_{3} \mathrm{O}_{4} @ \mathrm{Au}$ magnetic nanoparticles $\left(\mathrm{Fe}_{3} \mathrm{O}_{4} @ \mathrm{Au}\right.$ C225 composite-targeted MNPs) and utilized as a drug nanocarrier to perform targeted magneto-photothermal therapy against glioma cells [98]. The core-shell $\mathrm{Fe}_{3} \mathrm{O}_{4} @ \mathrm{Au}$ magnetic nanoparticles were then conjugated with antibodies, C225 make $\mathrm{Fe}_{3} \mathrm{O}_{4} @ \mathrm{Au}-\mathrm{C} 225$ composite-targeted MNPs. The rate of tumor inhibition and degree of apoptotic shown by $\mathrm{Fe}_{3} \mathrm{O}_{4} @ \mathrm{Au}-\mathrm{C} 225$ composite-targeted MNPs combined with hyperthermia (MFH + NIR) group were significantly higher than other groups in vitro and scored up-regulation of caspase-3, caspase-8, and caspase- 9 expression revealed excellent anti-tumor effect. Further, $\mathrm{Fe}_{3} \mathrm{O}_{4} @ \mathrm{Au}-\mathrm{C} 225$ composite-targeted MNPs-mediated combined hyperthermia (MFH + NIR) group demonstrated significant tumor growth suppression compared with other groups in vivo. Considering targeted chemotherapy and diagnosis of glial tumor simultaneously, Shevtsov and Colleagues prepared a hybrid chitosan-dextran superparamagnetic nanoparticle (CS-DX-MNPs) [99]. Confocal microscopic studies and flow cytometry confirmed the prompted cell internalization hybrid MNPs in U87, C6 glioma, and HeLa cells compared to dextran coated MNPs. Cytotoxicity revealed that toxic concentration synthesized hybrid MNPs was $10 \mu \mathrm{g} / \mathrm{ml}$. The MRI scanning and histopathological study confirmed uniform accumulation and retention inside the cells of i.v given MNPs in orthotopic C6 gliomas in rats. Therefore, hybrid chitosan-dextran MNPs demonstrated enhanced magnetic resonance contrast for delineation in the glial tumor. Further, owing to the plentiful retention of CS-DX-MNPs in the tumor, the application is not limited to tumor imaging but extends to the precise targeting of chemotherapeutics. In another study, carmustine loaded polyvinyl alcohol/polyethyleneimine/fluorescein isothiocyanate complex coated MNPs were developed with enhanced structural and chemical stability for site-specific delivery [100]. A path tracking agent fluorescein isothiocyanate conjugated to the NPs surface, which helps in cell internalization under the influence of the magnetic field. The resulting nanoparticle inhibited the growth of GBM up to $92.7 \%$ compared to plain carmustine.

\section{Carbon nanotubes}

Carbon nanotubes (CNTs) are a sheet of graphene molecules involutes in a cylindrical shape that constitutes a greatly valued nanomaterial with diverse physicochemical properties for range application in biomedical engineering [101]. The network-like structure of carbon generally insoluble in aqueous medium and length as well as the diameter of the tube varies depending upon the arrangement of graphene molecules. It possesses a large surface area for a high payload of multiple therapeutics anchored with the wall of the nanotube, and unique optical and mechanical properties. Surface modification renders them an ideal candidate for improving aqueous solubility, penetrating the ability of BBB, pH-dependent therapeutic release, biocompatibility, and minimizing toxicity as well [102]. In this context, Lu et al. developed FA conjugated DOX loaded multi-walled CNTs (FA-DOX-MWCNTs) and evaluated their therapeutic potential against U87 cells [103]. The developed FADOX-MWCNTs exhibited significantly enhanced cellular internalization in $\mathrm{U} 87$ cells due to receptor-mediated endocytosis, thereby improved cytotoxicity compared to non-targeted DOX-MWCNTs and free DOX. In another study, Romano-Feinholz and colleagues characterize the molecular geometry and biocompatibility aspect of different multi-walled carbon nanotubes (MWCNTs) in rat astrocytes and RG2 glioma [104]. They developed nitrogen-doped N-MWCNTs with the help of ferrocene, and the benzylamine pyrolysis technique followed aerosol-assisted chemical vapor deposition and functionalized. Further, they assessed the process of cell lysis induction and potential enhance therapeutic efficacy by the inclusion of TMZ in MWCNTs. The average diameter of pristine MWCNTs and pristine N-MWCNTs showed the particle size of $\sim 22$ and $\sim 35 \mathrm{~nm}$, respectively. The cell viability study confirmed the concentration-dependent cell death, and the lowest concentration reported $10 \mu \mathrm{g} / \mathrm{mL}$. Both in vitro and in vivo outcome intimated that these MWCNTs could be used as adjuvant therapy with standard treatment to enhance further the survival in malignant glioma bearing rats.

The findings of various preclinical investigations are providing evidence in support of better management of GBM exploiting different types of nanoparticles and outcomes summarized in > Table 1.

\section{Nanoparticles Based Gene Therapy of GBM}

Gene therapy is an energizing technique involves the therapeutic release of gene adequately contribute apoptosis while expressed in tumor cells. It involves both viral vectors and nonviral vectors through nanoparticle encapsulation. The cationic nanoparticle carrier composed of polyamino ester demonstrated increased transfection efficiency and lower cell toxicity. The cationic polymer merged with DNA could be capable of encapsulating several plasmids. The developed nanoparticles were analyzed for eradicating 
- Table 1 Smart nanoparticles for targeted treatment of GBM.

\begin{tabular}{|c|c|c|c|c|c|}
\hline Nanomedicine & Bioactive & Ligand & Animal/Cell line & Outcome & Ref \\
\hline Liposome & Doxorubicin (DOX) & $\begin{array}{l}\text { Angiopep-2, T7, } \\
\text { c(RGDfK), } \\
\text { Peptide-22, D-SP5 } \\
\text { and Pep-1. }\end{array}$ & $\begin{array}{l}\text { BALB/c mice/ } \\
\text { HUVECs and U87 } \\
\text { cells }\end{array}$ & $\begin{array}{l}\text { Higher cellular uptake and cytotoxicity, brain } \\
\text { distribution, and mean survival time compared to } \\
\text { single ligand decorated liposome and non-targeted } \\
\text { liposome }\end{array}$ & [60] \\
\hline Liposome & DOX & Transferrin (Tf) & HUVECs and C6 cells & $\begin{array}{l}\text { Higher cellular uptake via receptor-mediated } \\
\text { endocytosis and cytotoxicity compared to } \\
\text { non-targeted liposome and pure drug }\end{array}$ & [61] \\
\hline Liposome & DOX and erlotinib & Tf & $\begin{array}{l}\text { BALB/c mice/U87, } \\
\text { bEnd. } 3 \text { and glial } \\
\text { cells }\end{array}$ & $\begin{array}{l}\text { Higher in vitro cellular uptake and cytotoxicity } \\
\text { compared to the non-targeted liposome. } \\
\text { Biocompatible and showed higher brain uptake and } \\
\text { higher anti-tumor efficacy }\end{array}$ & {$[62]$} \\
\hline SLNs & Apolipoprotein E & Biotin & hCMEC/D3 cells & $\begin{array}{l}\text { Higher receptor-mediated endocytosis and } 1.5 \text {-fold } \\
\text { increment brain permeability compared to } \\
\text { non-biotinylated SLNs }\end{array}$ & [65] \\
\hline SLNs & Asiatic acid (AA) & cRGD & $\begin{array}{l}\text { BALB/c mice/ } \\
\text { U87-MG cells }\end{array}$ & $\begin{array}{l}\text { Higher cellular uptake and cytotoxicity compared to } \\
\text { non-targeted SLNs and pure drug. Significantly } \\
\text { higher tumor growth inhibition compared to } \\
\text { non-targeted SLNs and pure drug. }\end{array}$ & [66] \\
\hline SLNs & Indirubin (IN) & - & U87-MG cells & $\begin{array}{l}\text { Sustained release of drug from SLNs. Significantly } \\
\text { improved cellular uptake and cytotoxicity compared } \\
\text { to pure drug. }\end{array}$ & [67] \\
\hline NLCs & $\begin{array}{l}\text { Temozolomide } \\
\text { (TMZ) }\end{array}$ & cRGD & $\begin{array}{l}\text { BALB/c mice/ } \\
\text { U87MG cells }\end{array}$ & $\begin{array}{l}2 \text { and } 10 \text { folds reduction in } \mathrm{IC}_{50} \text { value compared to } \\
\text { non-targeted NLCs and } \mathrm{TMZ} \text { solution respectively. } \\
\text { Furthermore, higher tumor inhibition compared to } \\
\text { non-targeted NLCs and pure TMZ. }\end{array}$ & [71] \\
\hline NLCs & $\begin{array}{l}\text { TMZ and } \\
\text { Vincristine (VCR) }\end{array}$ & $\begin{array}{l}\text { Lactoferrin (Lf) and } \\
\text { CRGD }\end{array}$ & $\begin{array}{l}\text { BALB/c mice/ } \\
\text { U87MG cells }\end{array}$ & $\begin{array}{l}\text { Higher cellular uptake and cytotoxicity against U87 } \\
\text { MG cells compared to single ligand decorated NLCs, } \\
\text { non-targeted NLCs, and pure TMZ and VCR. Similar } \\
\text { results were found in in-vivo studies. }\end{array}$ & [72] \\
\hline NLCs & Curcumin (CUR) & - & $\begin{array}{l}\text { BALB/c mice/A172 } \\
\text { cells }\end{array}$ & $\begin{array}{l}\text { Significantly improved cellular uptake and about } 4 \\
\text { fold reduction in } \mathrm{IC}_{50} \text { value compared to pure CUR. } \\
\text { Furthermore, }\end{array}$ & [73] \\
\hline Polymeric NPs & TMZ & $\begin{array}{l}\text { Monoclonal } \\
\text { antibody (mAb) }\end{array}$ & U251 and U87 cells & $\begin{array}{l}\text { Significantly higher cellular uptake and cytotoxicity } \\
\text { compared to non-targeted NPs and pre TMZ. }\end{array}$ & [79] \\
\hline Polymeric NPs & Shikonin (SHK) & Lf & $\begin{array}{l}\text { BALB/c mice/C6 } \\
\text { cells }\end{array}$ & $\begin{array}{l}\text { Higher cellular uptake and } 1.2 \text { and } 1.43 \text { fold } \\
\text { reduction in IC } C_{50} \text { value compared to non-targeted } \\
\text { NPs and pure SHK, respectively. }\end{array}$ & [80] \\
\hline Polymeric NPs & Melatonin (MLT) & - & $\begin{array}{l}\text { Wistar rats/U87MG } \\
\text { cells }\end{array}$ & $\begin{array}{l}\text { Higher cellular uptake and } 2500 \text { fold reduction in } \\
\mathrm{IC}_{50} \text { compared to MLT solution. Furthermore, } 7.6 \\
\text { fold improved absolute bioavailability after } \\
\text { intranasal administration compared to MLT } \\
\text { suspension. }\end{array}$ & [81] \\
\hline Polymeric micelles & Epirubicin (Epi) & cRGD & U87MG cells & $\begin{array}{l}\text { Significantly higher cellular uptake by integrin } \\
\text { receptor-mediated endocytosis and cytotoxicity } \\
\text { compared to non-targeted micelles and pre EPI }\end{array}$ & [84] \\
\hline Polymeric micelles & Docetaxel (DTX) & cRGD & $\begin{array}{l}\text { BALB/c mice/ } \\
\text { U87MG cells }\end{array}$ & $\begin{array}{l}\text { Significantly enhanced cellular uptake and } \\
\text { cytotoxicity compared to non-targeted micelles and } \\
\text { pure DTX. Higher tumor uptake through integrin } \\
\text { receptor-mediated endocytosis and anti-glioma } \\
\text { efficacy compared to non-targeted micelles and } \\
\text { pure DTX. }\end{array}$ & [85] \\
\hline Polymeric micelles & $\begin{array}{l}\text { DOX and Paclitaxel } \\
\text { (PTX) }\end{array}$ & cRGD & $\begin{array}{l}\text { BALB/c mice/ } \\
\text { U87MG cells }\end{array}$ & $\begin{array}{l}\text { Significantly higher transport rates across the } \\
\text { in vitro blood-brain barrier model, cellular internaliza- } \\
\text { tion, cytotoxicity, and apoptosis compared to } \\
\text { non-targeted micelles and native drugs. Further, } \\
\text { targeted micelles exhibited significantly higher } \\
\text { tumor accumulation and tumor inhibition rates } \\
\text { compared to non-targeted micelles and native } \\
\text { drugs. }\end{array}$ & [86] \\
\hline
\end{tabular}


- Table 1 Continued.

\begin{tabular}{|c|c|c|c|c|c|}
\hline Nanomedicine & Bioactive & Ligand & Animal/Cell line & Outcome & Ref \\
\hline Dendrimers & DTX & $\mathrm{pHBA}$ & U87MG cells & $\begin{array}{l}\text { Significantly improved cellular uptake by sigma } \\
\text { receptor-mediated endocytosis and cytotoxicity } \\
\text { compared to non-targeted dendrimers and } \\
\text { marketed preparation Taxotere }{ }^{\circledR} \text {. }\end{array}$ & [89] \\
\hline Dendrimers & TMZ & - & $\begin{array}{l}\text { U-251 and T-98G } \\
\text { cells }\end{array}$ & $\begin{array}{l}\text { Significantly improved cellular uptake and } \\
\text { cytotoxicity compared to pure TMZ. Furthermore, } \\
1.5 \text { fold improved brain bioavailability compared to } \\
\text { free TMZ. }\end{array}$ & {$[90]$} \\
\hline Dendrimers & DOX & - & $\begin{array}{l}\text { Sprague Dawley } \\
\text { rats/U87 MG cells }\end{array}$ & $\begin{array}{l}\text { Significantly enhanced cellular uptake and } \\
\text { cytotoxicity compared to free DOX. Higher survival } \\
\text { rate compared to free DOX. }\end{array}$ & [91] \\
\hline $\begin{array}{l}\text { Gold nanoparticles } \\
\text { (GNPs) }\end{array}$ & SiRNA & - & U87MG cells & $\begin{array}{l}22 \% \text { reduction in } \mathrm{BCL}-2 \text { proteins level compared to } \\
\text { the native genetic material }\end{array}$ & [93] \\
\hline GNPs & miR-182 & - & $\begin{array}{l}\text { U87 cells and } \\
\text { patient-derived } \\
\text { glioma-initiating } \\
\text { stem-like cells } \\
\text { (GICs) }\end{array}$ & $\begin{array}{l}\text { Significantly decrease in miR } 182 \text { target genes } \\
\text { BCL2L12 and c-Met protein levels compared to } \\
\text { native genetic material. Similar results were found } \\
\text { in in-vivo studies. }\end{array}$ & {$[94]$} \\
\hline $\begin{array}{l}\text { Magnetic nanoparti- } \\
\text { cles (MNPs) }\end{array}$ & - & Cetuximab & $\begin{array}{l}\text { Balb/c nu/nu nude } \\
\text { mice/U251 cells }\end{array}$ & $\begin{array}{l}\text { MNPs combined with hyperthermia (MFH+NIR) } \\
\text { group were significantly higher apoptosis compared } \\
\text { to other groups in vitro and scored up-regulation of } \\
\text { caspase-3, caspase-8, and caspase- } 9 \text { expression. } \\
\text { Further, MNPs-mediated combined hyperthermia } \\
\text { (MFH+NIR) group demonstrated significant tumor } \\
\text { growth suppression compared with other groups } \\
\text { in vivo. }\end{array}$ & [98] \\
\hline Dextran coated MNPs & - & Dextran & $\begin{array}{l}\text { Male Wistar rats/ } \\
\text { U87, C6, and HeLa } \\
\text { cells }\end{array}$ & $\begin{array}{l}\text { Significantly higher cellular uptake and cytotoxicity } \\
\text { compared to native NPs. MRI scanning and the } \\
\text { histopathological study confirmed uniform } \\
\text { accumulation and retention inside the tumor after } \\
\text { i.v administration. Further, the developed MNPs } \\
\text { exhibited significantly higher tumor inhibition in } \\
\text { rats compared to other groups. }\end{array}$ & [99] \\
\hline MNPs & Carmustine & & A172 cells & $\begin{array}{l}\text { Significantly higher cellular uptake and cytotoxicity } \\
\text { compared to free drug. }\end{array}$ & {$[100]$} \\
\hline $\begin{array}{l}\text { Multi-walled carbon } \\
\text { nanotubes (MWCNTs) }\end{array}$ & DOX & $\mathrm{FA}$ & U87 cells & $\begin{array}{l}\text { Significantly higher cellular uptake due to } \\
\text { receptor-mediated endocytosis and cytotoxicity } \\
\text { compared to non-targeted MWCNTs and free drug. }\end{array}$ & [103] \\
\hline MWCNTS & TMZ & - & $\begin{array}{l}\text { Fischer } 344 \text { male } \\
\text { rats/RG2 cells }\end{array}$ & $\begin{array}{l}\text { The developed MWCNTs significantly higher cellular } \\
\text { uptake and induction of apoptosis through G2-M } \\
\text { phase cell cycle arrest. }\end{array}$ & [104] \\
\hline
\end{tabular}

glioma cells in the brain, and the result demonstrated that higher transfection efficiency in GBM and astrocytes. The in vivo study of cationic DNA nanoparticles showed the highest transfection efficiency upon injection into tumor-bearing mice compared to naked DNA [105, 106]. Nanotechnology-mediated RNA interference (RNAi) has shown significant potential to overcome the challenges associated with the conventional treatment approach for GBM. In addition, the complexity of the brain physiology simply enhanced penetration to BBB or approached for tumor-targeting cannot exclusively meet the atypical needs of various therapeutic delivery stages. In a study, a charge conversational biomimetic nanocomplex was developed with a three-layer core-shell structure to systematically overcome the consistent hurdles in siRNA delivery to GBM [107]. The designing of such nanocomplex provides good biocompatibility along with prolonged blood circulation, high BBB transcytosis, and effective tumor accumulation, and specifically uptake by the brain tumor cells as well as its effective accumulation in tumor tissues. Furthermore, red blood cell membrane (RBCm) disruption, as well as the effective release of siRNA can be further elicited elegantly by charge transformation from negative to positive in the endo/lysosome ( $\mathrm{pH}$ 5.0-6.5) of tumor cells. It is ultimately leading to highly significant target-gene silencing along with the strong anti-GBM activity. This investigation provides an intelligent biomimetic nanocomplex designed for systemic siRNA delivery to GBM, leveraging Angiopep-2 peptide-modified, immune-free $\mathrm{RBCm}$, and charge conversational components. Enhanced therapeutic efficacy, increase in survival rates, and minimization of systemic side effects was achieved in orthotopic tumor-bearing nude mice having U87MG-luc human glioblastoma. In another study, Janiszewska and coworkers developed poly(lysine) (PLL) dendrons for drug and siRNA nanocarriers owing to their low cytotoxicity, high biocompatibility, and capable of forming globular structures 
such as protein in aqueous media [108]. The anti-p42 MAPK siRNA loaded PLL dendrons ensued 35-40\% decrease in the levels of the p42-MAPK protein when transfecting in U87 and C6 glioma cells. However, the dendrons exhibited cytotoxicity in glioma cells, leaving cortical neurons or astrocytes unaffected, which comprised the healthy counterpart cells with GBM inside the brain. Hayward et al. developed FA conjugated pRNA loaded liposomes for targeted treatment of GBM [109]. Targeted liposomes exhibited significantly higher cellular uptake and cytotoxicity against U87EGFRvIll cells. Compared to non-targeted liposomes. Moreover, similar results were observed in an in vivio study in athymic female nu/nu mice bearing U87EGFRvIll xenograft. Very recently, Yang et al. developed RGD decorated PTX and siRNA co-loaded nanoparticles for synergistic efficacy against GBM [110]. The developed targeted nanoparticles exhibited significantly improved cellular uptake and cytotoxicity against $U 87$ cells compared to non-targeted nanoparticles. Moreover, in vivo anti-GBM efficacy study revealed significantly higher therapeutic efficacy of drug and siRNA co-loaded targeted nanoparticles in nude female BALB/c mice bearing U87 xenografts compared to non-targeted nanoparticles and single agents loaded nanoparticles.

\section{Future Prospects}

The contempt of wide search for GBM unmet challenges still on the way for targeted therapies of highly devastating GBM. The identification of critical molecular pathways, downstream and upstream signals directly involved in the development of GBM due to genetic mutation leads to tumor cell proliferation and survival essentials to be addressed. Due to the limited therapeutic efficacy of radiation therapy in combination with chemotherapeutic drugs, the use of functional nanoparticles as targeted therapy is highly desirable for effective and safe therapeutic delivery. Nevertheless, nanotechnology is a better platform for enriching therapeutic efficacy by chemical or surface alteration of smart nanoparticles followed by functionalization for receptor-mediated therapies for delivering therapeutics agents, gene delivery, and theranostic agents in GBM. The future of drug encapsulated nanoparticles required comprehensive knowledge of its physicochemical properties, surface characteristics, toxicity profile, biostability, biodistribution, clearance, and safety assessment will further push up therapeutic advances in GBM.

\section{Conclusion}

The blood-brain (primary) and blood-brain tumor (secondary) are the major obstacles in the path of drug delivery for therapeutic modalities of GBM. The anatomy of the brain is associated with such vital barrier confined drug delivery only to the particular arena of the brain and the main part of the brain getting deprived of therapeutic molecules. Hence, this makes a mandate to switch over to the alternate therapeutic approach in order to accomplish effective therapeutic concentration in brain tumor tissue. The ongoing shift from conventional therapy to targeted drug delivery to the brain gains primary attention nowadays due to more precise controlled drug release to the affected area. The recent advances in drug delivery to the brain as smart nanoparticles for targeted ther- apies have been ascertained for the therapeutic modality in GBM. The thorough understanding of molecular pathways involved in brain targeting and receptor-based therapeutic potential will further enrich the outcomes of the therapies. Nevertheless, most of the potential technology is under the developing stage, and continuing efforts inferring the underlying mechanism involved in drug delivery will faster translate the nanoparticles into clinical application. Moreover, combating such barriers for CNS drug delivery, several advanced nanoparticles have been developed so far, employing multifunctional smart nanoparticles, and their potential utility is clinically proven or under the trial phase.

\section{Acknowledgements}

Md. Habban Akhter would like to acknowledge the Faculty of Pharmacy, DIT University, Dehradun India, for furnishing a substantial platform for grooming of the manuscript.

\section{Conflicts of Interest}

The authors declare that they have no conflict of interest. The authors alone are responsible for the content and writing of this article.

\section{References}

[1] Fakhoury M. Drug delivery approaches for the treatment of glioblastoma multiforme. Artif Cells Nanomed Biotechnol 2016; 44: 1365-1373

[2] https://www.wcrf.org/dietandcancer/cancer-trends/worldwidecancer-data

[3] Zhou ], Atsina KB, Himes BT et al. Novel delivery strategies for glioblastoma. Cancer J 2012; 18: 89-99

[4] Arjona D, Rey JA, Taylor SM. Early genetic changes involved in low-grade astrocytic tumor development. Curr Mol Med 2006; 6: 645-650

[5] Parsons DW, Jones S, Zhang X et al. An integrated genomic analysis of human glioblastoma multiforme. Science 2008; 321: 1807-1812

[6] Jhanwar-Uniyal M, Labagnara M, Friedman M et al. Glioblastoma: Molecular pathways, stem cells and therapeutic targets. Cancers (Basel) 2015; 7: 538-555

[7] Ahmad J, Akhter S, Rizwanullah M et al. Nanotechnology based theranostic approaches in alzheimer's disease management: Current status and future perspective. Curr Alzheimer Res 2017; 14: 1164-1181

[8] Barkat MA, Harshita, Rizwanullah M et al. Therapeutic nanoemulsion: Concept to delivery. Curr Pharm Des 2020; 26: 1145-1166

[9] Ahmad J, Rizwanullah M, Amin S et al. Nanostructured lipid carriers (NLCs): Nose-to-brain delivery and theranostic application. Curr Drug Metab 2020; 21: 1-8. doi:10.2174/1389200221666200719003304

[10] Nam L, Coll C, Erthal LC et al. Drug delivery nanosystems for the localized treatment of glioblastoma multiforme. Materials 2018; 11 : 779. doi:10.3390/ma11050779

[11] Raucher D, Dragojevic S, Ryu J. Macromolecular drug carriers for targeted glioblastoma therapy: Preclinical studies, challenges, and future perspectives. Front Oncol 2018; 8: 624. doi:10.3389/ fonc. 2018.00624 
[12] Ozturk-Atar K, Eroglue H, Calis S. Novel advances in targeted drug delivery. J Drug Target 2018; 26: 633-642

[13] Kang C, Sun Y, Zhu Y et al. Delivery of nanoparticles for treatment of brain tumor. Curr Drug Metab 2016; 17: 745-754

[14] Kaisar MA, Sajja RK, Prasad S et al. New experimental models of the blood-brain barrier for CNS drug discovery. Expert Opin Drug Discov 2017; 12: 89-103

[15] Patel M, Souto EB, Singh KK. Advances in brain drug targeting and delivery: Limitations and challenges of solid lipid nanoparticles. Expert Opin Drug Deliv 2013; 10: 889-905

[16] Gao H. Progress and perspectives on targeting nanoparticles for brain drug delivery. Acta Pharm Sin B 2016; 6: 268-286

[17] Wang $X, Y u X$, Vaughan $W$ et al. Novel drug-delivery approaches to the blood-brain barrier. Neurosci Bull 2015; 31: 257-264

[18] Ronaldson PT, Davis TP. Targeting transporters: Promoting blood-brain barrier repair in response to oxidative stress injury. Brain Res 2015; 1623: 39-52

[19] Oller-Salvia B, Sanchez-Navarro M, Giraltab E et al. Blood-brain barrier shuttle peptides: an emerging paradigm for brain delivery. Chem Soc Rev 2016; 45: 4690-4707

[20] Gutkin A, Cohen ZR, Peer D. Harnessing nanomedicine for therapeutic intervention in glioblastoma. Expert Opin Drug Deliv 2016; 13: 1573-1582

[21] Grabrucker AM, Ruozi B, Belletti D et al. Nanoparticle transport across the blood brain barrier. Tissue Barriers 2016; 4: e1153568

[22] Banks WA. From blood-brain barrier to blood-brain interface: New opportunities for CNS drug delivery. Nat Rev Drug Discov 2016; 15: 275-292

[23] Pardridge WM. Drug transport across the blood-brain barrier. J Cereb Blood Flow Metab 2012; 32: 1959-1972

[24] Lichota J, Skjørringe T, Thomsen LB et al. Macromolecular drug transport into the brain using targeted therapy. J Neurochem 2010; 113: $1-13$

[25] Sagare AP, Deane R, Zlokovic BV. Low-density lipoprotein receptorrelated protein 1: A physiological $A \beta$ homeostatic mechanism with multiple therapeutic opportunities. Pharmacol Ther 2012; 136: 94-105

[26] Yu Y], Zhang Y, Kenrick M et al. Boosting brain uptake of a therapeutic antibody by reducing its affinity for a transcytosis target. Sci Transl Med 2011; 3: 84ra44

[27] Miura Y, Takenaka T, Toh K. Cyclic RGD-linked polymeric micelles for targeted delivery of platinum anticancer drugs to glioblastoma through the blood-brain tumor barrier. ACS Nano 2013; 7 : 8583-8592

[28] Demeule M, Currie JC, Bertrand Y et al. Involvement of the low-density lipoprotein receptor-related protein in the transcytosis of the brain delivery vector angiopep-2. J Neurochem 2008; 106: 1534-1544

[29] ClinicalTrials.gov identifier: NCT01480583

[30] Rao KS, Reddy MK, Horning JL et al. TAT-conjugated nanoparticles for the CNS delivery of anti-HIV drugs. Biomaterials 2008; 29: 44294438

[31] Gao H, Zhang S, Cao S et al. Angiopep-2 and activatable cell-penetrating peptide dual-functionalized nanoparticles for systemic glioma-targeting delivery. Mol Pharm 2014; 11: 2755-2763

[32] Cancer Genome Atlas Research Network, Comprehensive genomic characterization defines human glioblastoma genes and core pathways. Nature 2008; 455: 1061-1068

[33] Pearson JRD, Regad T. Targeting cellular pathways in glioblastoma multiforme. Signal Transduct Target Ther 2017; 2: e17040

[34] Khan MA, Jain VK, Rizwanullah M et al. PI3K/AKT/mTOR pathway inhibitors in triple-negative breast cancer: A review on drug discovery and future challenges. Drug Discov Today 2019; 24: 2181-2191
[35] Regad T, Targeting RTK. signaling pathways in cancer. Cancers (Basel) 2015; 7: 1758-1784

[36] Joensuu $H$, Puputti M, Sihto $H$ et al. Amplification of genes encoding KIT, PDGFR $\alpha$ and VEGFR2 receptor tyrosine kinases is frequent in glioblastoma multiforme. J Pathol 2005; 207: 224-231

[37] Akhter MH, Madhav NVS, Ahmad J. Epidermal growth factor based active targeting: A paradigm shift towards advance tumor therapy. Artif Cells Nanomed Biotechnol 2018; 46: 1188-1198

[38] Polivka J Jr, Janku F. Molecular targets for cancer therapy in the PI3K/ AKT/mTOR pathway. Pharmacol Ther 2014; 142: 164-175

[39] Carracedo A, Pandolfi PP. The PTEN-PI3K pathway: of feedbacks and cross-talks. Oncogene 2008; 27: 5527-5541

[40] Prasad G, Sottero T, Yang X et al. Inhibition of PI3K/mTOR pathways in glioblastoma and implications for combination therapy with temozolomide. Neuro Oncol 2011; 13: 384-392

[41] Masri J, Bernath A, Martin J et al. mTORC2 activity is elevated in gliomas and promotes growth and cell motility via overexpression of rictor. Cancer Res 2007; 67: 11712-11720

[42] He G, Siddik ZH, Huang Z et al. Induction of p21 by p53 following DNA damage inhibits both Cdk4 and Cdk2 activities. Oncogene 2005; 24: 2929-2943

[43] Michaud K, Solomon DA, Oermann E et al. Pharmacologic inhibition of cyclin-dependent kinases 4 and 6 arrests the growth of glioblastoma multiforme intracranial xenografts. Cancer Res 2010; 70: 3228-3238

[44] Han J, Alvarez-Breckenridge CA, Wang QE et al. $\beta$ signaling and its targeting for glioma treatment. Am J Cancer Res 2015; 5: 945-955

[45] Lamouille S, Derynck R. Cell size and invasion in TGF-beta-induced epithelial to mesenchymal transition is regulated by activation of the mTOR pathway. J Cell Biol 2007; 178: 437-451

[46] Rooke HM, Crosier KE. The smad proteins and TGF beta signaling: uncovering a pathway critical in cancer. Pathology 2001; 33: 73-84

[47] Petterson SA, Dahlrot RH, Hermansen SK et al. High levels of c-Met is associated with poor prognosis in glioblastoma. J Neurooncol 2015; 122: $517-527$

[48] Cruickshanks N, Zhang Y, Yuan F et al. Role and therapeutic targeting of the HGF/MET pathway in glioblastoma. Cancers (Basel) 2017; 9: e87

[49] Brennan CW, Verhaak RG, McKenna A et al. The somatic genomic landscape of glioblastoma. Cell 2013; 155: 462-477

[50] Sayal KK, Higgins GS, Hammond EM. Uncovering the influence of the FGFR1 pathway on glioblastoma radiosensitivity. Ann Transl Med 2016; 4: e538

[51] Gouazé-Andersson V, Delmas C, Taurand M et al. FGFR1 induces glioblastoma radioresistance through the PLCY/Hif1 $\alpha$ pathway. Cancer Res 2016; 76: 3036-3044

[52] Maris C, D'Haene N, Trépant AL et al. IGF-IR: a new prognostic biomarker for human glioblastoma. British J Cancer 2015; 113: 729-737

[53] Leclerc C, Haeich J, Aulestia F] et al. Calcium signaling orchestrates glioblastoma development: Facts and conjunctures. Biochim Biophys Acta 2016; 1863: 1447-1459

[54] Zhang Y, Cruickshanks N, Yuan F et al. Targetable T-type calcium channels drive glioblastoma. Cancer Res 2017; 77: 3479-3490

[55] Fajardo AM, Piazza GA, Tinsley HN. The role of cyclic nucleotide signaling pathways in cancer: targets for prevention and treatment. Cancers 2014; 6: 436-458

[56] Fiscus RR. Involvement of cyclic GMP and protein kinase G in the regulation of apoptosis and survival in neural cells. Neurosignals 2002; 11: 175-190 
[57] Mahtab A, Rizwanullah M, Pandey S et al. Quality by design driven development and optimization of Teriflunomide loaded nanoliposomes for treatment of rheumatoid arthritis: An in vitro and in vivo assessments. J Drug Deliv Sci Tech 2019; 51: 383-396

[58] Gilani S], Jahangir MA, Rizwanullah M et al. Nano-based therapy for treatment of skin cancer. Recent Pat Antiinfect Drug Discov 2018; 13 : $151-163$

[59] Deshpande PP, Biswas S, Torchilin VP. Current trends in the use of liposomes for tumor targeting. Nanomedicine 2013; 8: 1509-1528

[60] Chen C, Duan Z, Yuan Y et al. Peptide-22 and Cyclic RGD functionalized liposomes for glioma targeting drug delivery overcoming BBB and BBTB. ACS Appl Mater Interfaces 2017; 9: 5864-5873

[61] Liu C, Liu XN, Wang GL et al. A dual-mediated liposomal drug delivery system targeting the brain: Rational construction, integrity evaluation across the blood-brain barrier, and the transporting mechanism to glioma cells. Int ] Nanomedicine 2017; 12: 2407-2425

[62] Lakkadwala S, Dos Santos Rodrigues B, Sun C et al. Dual functionalized liposomes for efficient co-delivery of anticancer chemotherapeutics for the treatment of glioblastoma. J Control Release 2019; 307 : 247-260

[63] Rizwanullah M, Amin S, Ahmad ]. Improved pharmacokinetics and antihyperlipidemic efficacy of rosuvastatin-loaded nanostructured lipid carriers. J Drug Target 2017; 25: 58-74

[64] Varghese NM, Venkatachalam S, Saxena SK. Nanocarriers for brain specific delivery of antiretro viral drugs: Challenges and achievements. J Drug Target 2018; 26: 195-207

[65] Neves AR, Queiroz JF, Weksler B et al. Solid lipid nanoparticles as a vehicle for brain-targeted drug delivery: Two new strategies of functionalization with apolipoprotein E. Nanotechology 2015; 26: 495103

[66] Garanti T, Alhnan MA, Wan KW. RGD-decorated solid lipid nanoparticles enhanced tumor targeting, penetration and anticancer effect of asiatic acid. Nanomedicine (Lond) 2020; 15: 1567-1583

[67] Rahiminejad A, Dinarvand R, Johari B et al. Preparation and investigation of indirubin-loaded SLN nanoparticles and their anticancer effects on human glioblastoma U87MG cells. Cell Biol Int 2019; 43: 2-11

[68] Soni K, Rizwanullah M, Kohli K. Development and optimization of sulforaphane-loaded nanostructured lipid carriers by the BoxBehnken design for improved oral efficacy against cancer: in vitro, ex vivo and in vivo assessments. Artif Cells Nanomed Biotechnol 2018; 46: 15-31

[69] Harshita, Barkat MA, Rizwanullah M et al. Paclitaxel-loaded nanolipidic carriers with improved oral bioavailability and anticancer activity against human liver carcinoma. AAPS PharmSciTech 2019; 20: e87

[70] Rizwanullah M, Ahmad J, Amin S. Nanostructured lipid carriers: A novel platform for chemotherapeutics. Curr Drug Deliv 2016; 13: 4-26

[71] Song S, Mao G, Du J et al. Novel RGD containing, temozolomideloading nanostructured lipid carriers for glioblastoma multiforme chemotherapy. Drug Deliv 2016; 23: 1404-1408

[72] Zhang J, Xiao X, Zhu J et al. Lactoferrin- and RGD-comodified, temozolomide and vincristine-coloaded nanostructured lipid carriers for gliomatosis cerebri combination therapy. Int J Nanomedicine 2018; 13: 3039-3051

[73] Chen Y, Pan L, jiang $M$ et al. Nanostructured lipid carriers enhance the bioavailability and brain cancer inhibitory efficacy of curcumin both in vitro and in vivo. Drug Deliv 2016; 23: 1383-1392

[74] Rizwanullah M, Amin S, Mir SR et al. Phytochemical based nanomedicines against cancer: Current status and future prospects. J Drug Target 2018; 26: 731-752
75] Akhter MH, Rizwanullah M, Ahmad J et al. Nanocarriers in advanced drug targeting: Setting novel paradigm in cancer therapeutics. Artif Cells Nanomed Biotechnol 2018; 46: 873-884

[76] Shahab MS, Rizwanullah M, Alshehri S et al. Optimization to development of chitosan decorated polycaprolactone nanoparticles for improved ocular delivery of dorzolamide: In vitro, ex vivo and toxicity assessments. Int ] Biol Macromol 2020; 163: 2392-2404

[77] Rizwanullah M, Alam M, Harshita et al. Polymer-lipid hybrid nanoparticles: A next-generation nanocarrier for targeted treatment of solid tumors. Curr Pharm Des 2020; 26: 1206-1215

[78] Khan FA, Rizwanullah M, Perwez A et al. Polymeric Nanomedicines in Treatment of Breast Cancer: Review of Contemporary Research. Frontiers in Anti-Cancer Drug Discovery. 2020; 11: 36-66

[79] Ramalho MJ, Sevin E, Gosselet F et al. Pereira, Receptor-mediated PLGA nanoparticles for glioblastoma multiforme treatment. Int ] Pharm 2018; 545: 84-92

[80] Li H, Tong Y, Bai L et al. Lactoferrin functionalized PEG-PLGA nanoparticles of shikonin for brain targeting therapy of glioma. Int ] Biol Macromol 2018; 107: 204-211

[81] de Oliveira RR Jr, Nascimento TL, Salomão MA et al. Increased nose-to-brain delivery of melatonin mediated by polycaprolactone nanoparticles for the treatment of glioblastoma. Pharm Res 2019; 36: e131

[82] Morshed RA, Cheng Y, Auffinger B et al. The potential of polymeric micelles in the context of glioblastoma therapy. Front Pharmacol 2013; 4: e157

[83] Haider N, Fatima S, Taha M et al. Nanomedicines in diagnosis and treatment of cancer: An update. Curr Pharm Des 2020; 26: 1216-1231

[84] Quader S, Liu X, Chen Y et al. cRGD peptide-installed epirubicinloaded polymeric micelles for effective targeted therapy against brain tumors. J Control Release 2017; 258: 56-66

[85] Li AJ, Zheng HY, Liu GD et al. Efficient delivery of docetaxel for the treatment of brain tumors by cyclic RGD-tagged polymeric micelles. Mol Med Rep 2015; 11: 3078-3086

[86] Huang Y, Liu W, Gao F et al. c(RGDyK)-decorated Pluronic micelles for enhanced doxorubicin and paclitaxel delivery to brain glioma. Int ] Nanomedicine 2016; 11: 1629-1641

[87] Kannan RM, Nance E, Kannan S et al. Emerging concepts in dendrimer-based nanomedicine: from design principles to clinical applications. J Intern Med 2014; 276: 579-617

[88] Akhter MH, Ahsan M], Rahman M, Anwar S, Rizwanullah M et al. Advancement in nanotheranostics for effective skin cancer therapy: State of the art. Curr Nanomed 2020; 10: 90-104

[89] Swami R, Singh I, Kulhari H et al. P-hydroxy benzoic acid conjugated dendrimer nanotherapeutics as potential carriers for targeted drug delivery to brain: An in vitro and in vivo evaluation. J Nanoparticle Res 2015; 17: e265

[90] Sharma AK, Gupta L, Sahu H et al. Chitosan engineered PAMAM dendrimers as nanoconstructs for the enhanced anticancer potential and improved in vivo brain pharmacokinetics of temozolomide. Pharm Res 2018; 35: e9

[91] Gajbhiye V, Jain NK. The treatment of glioblastoma xenografts by surfactant conjugated dendritic nanoconjugates. Biomaterials 2011; 32: 6213-6225

[92] Pinel S, Thomas N, Boura C et al. Approaches to physical stimulation of metallic nanoparticles for glioblastoma treatment. Adv Drug Deliv Rev 2019; 138: 344-357

[93] Kong L, Qiu J, Sun W et al. Multifunctional PEl-entrapped gold nanoparticles enable efficient delivery of therapeutic siRNA into glioblastoma cells. Biomater Sci 2017; 5: 258-266 
[94] Kouri FM, Hurley LA, Daniel WL et al. miR-182 integrates apoptosis, growth, and differentiation programs in glioblastoma. Genes Dev 2015; 29: 732-745

[95] Zhao L, Li Y, Zhu J et al. Chlorotoxin peptide-functionalized polyethylenimine-entrapped gold nanoparticles for glioma SPECT/CT imaging and radionuclide therapy. J Nanobiotechnology 2019; 17: e30

[96] Wankhede M, Bouras A, Kaluzova M et al. Magnetic nanoparticles: an emerging technology for malignant brain tumor imaging and therapy. Expert Rev Clin Pharmacol 2012; 5: 173-186

[97] Mahmoudi K, Bouras A, Bozec D et al. Magnetic hyperthermia therapy for the treatment of glioblastoma: A review of the therapy's history, efficacy and application in humans. Int J Hyperthermia 2018; 34: 1316-1328

[98] Lu Q, Dai X, Zhang P et al. Fe304@Au composite magnetic nanoparticles modified with cetuximab for targeted magneto-photothermal therapy of glioma cells. Int J Nanomedicine 2018; 13 : 2491-2505

[99] Shevtsov M, Nikolaev B, Marchenko Y et al. Targeting experimental orthotopic glioblastoma with chitosan-based superparamagnetic iron oxide nanoparticles (CS-DX-SPIONs). Int J Nanomedicine 2018; 13: $1471-1482$

[100] Akilo OD, Choonara YE, Strydom AM et al. An in vitro evaluation of a carmustine-loaded Nano-co-Plex for potential magnetic-targeted intranasal delivery to the brain. Int J Pharm 2016; 500: 196-209

[101] Kafa H, Wang JTW, Al-Jamal KT. Current perspective of carbon nanotubes application in neurology. Int Rev Neurobiol 2016; 130: 229-263
[102] Guo Q, Shen XT, Li YY et al. Carbon nanotubes-based drug delivery to cancer and brain. J Huazhong Univ Sci Technol Med Sci 2017; 37 : 635-641

[103] Lu Y], Wei KC, Ma CCM et al. Dual targeted delivery of doxorubicin to cancer cells using folate-conjugated magnetic multi-walled carbon nanotubes. Colloids Surf B Biointerfaces 2012; 89: 1-9

[104] Romano-Feinholz S, Salazar-Ramiro A, Muñoz-Sandoval E et al. Cytotoxicity induced by carbon nanotubes in experimental malignant glioma. Int J Nanomedicine 2017; 12: 6005-6026

[105] Pottoo FH, Javed MN, Rahman JU et al. Targeted delivery of miRNA based therapeuticals in the clinical management of Glioblastoma Multiforme. Semin Cancer Biol 2020, doi:10.1016/j.semcancer.2020.04.001

[106] Mangraviti A, Tzeng SY, Kozielski KL et al. Polymeric nanoparticles for nonviral gene therapy extend brain tumor survival in vivo. ACS Nano 2015; 9: 1236-1249

[107] Liu Y, Zou Y, Feng C et al. Charge conversional biomimetic nanocomplexes as a multifunctional platform for boosting orthotopic Glioblastoma RNAi therapy. Nano Lett 2020; 20: 1637-1646

[108] Janiszewska J, Posadas I, Játiva P et al. Second generation amphiphilic poly-lysine dendrons inhibit glioblastoma cell proliferation without toxicity for neurons or astrocytes. PLoS One 2016; 11: e0165704

[109] Hayward SL, Wilson CL, Kidambi S. Hyaluronic acid-conjugated liposome nanoparticles for targeted delivery to CD44 overexpressing glioblastoma cells. Oncotarget 2016; 7: 34158-34171

[110] Yang J, Zhang Q, Liu Y et al. Nanoparticle-based co-delivery of siRNA and paclitaxel for dual-targeting of glioblastoma. Nanomedicine(Lond) 2020; 15: 1391-1409 